\title{
Downside Risk of Derivative Portfolios with Mean-Reverting Underlyings
}

\author{
by
}

Patrick L. Leoni

Discussion Papers on Business and Economics

No. 2/2009

FURTHER INFORMATION

Department of Business and Economics

Faculty of Social Sciences

University of Southern Denmark

Campusvej 55

DK-5230 Odense M

Denmark

Tel.: +4565503271

Fax: +4565503237

E-mail: lho@sam.sdu.dk

ISBN 978-87-91657-30-6

http://www.sdu.dk/osbec 


\title{
Downside Risk of Derivative Portfolios with Mean-Reverting Underlyings
}

\author{
Patrick L. Leoni*
}

\begin{abstract}
We carry out a Monte-Carlo simulation of a standard portfolio management strategy involving derivatives, to estimate the sensitivity of its downside risk to a change of mean-reversion of the underlyings. We find that the higher the intensity of mean-reversion, the lower the probability of reaching a pre-determined loss level. This phenomenon appears of large statistical significance for large enough loss levels. We also find that the higher the mean-reversion intensity of the underlyings, the longer the expected time to reach those loss levels. The simulations suggest that selecting underlyings with high mean-reversion effect is a natural way to reduce the downside risk of those widely traded assets.
\end{abstract}

*University of Southern Denmark, Department of Business and Economics, Campusvej 55 DK-5230 Odense M, Denmark. E-mail: pal@sam.sdu.dk 


\section{Introduction}

Most of the worst financial disasters since the 70s have been caused by derivatives. For instance in the early 90s, Barings Bank lost $\$ 1$ bn after dubious trades with interest rates futures, causing in turn the bankruptcy of this well-established bank. In 1998, Long Term Capital Management lost \$4bn on somewhat similar products resulting as well in bankruptcy. More recently in January 2008, the French bank Société Générale realized a record loss of $\$ 7.1 \mathrm{bn}$ after dubious trades on standard derivatives. Given the severe losses incurred with those products, and their always increasing volume of trades, both practitioners and regulators have sought managerial techniques to reduce the downside of derivative portfolios.

In practice, the common strategy to reduce and/or to control the downside risk of derivatives is to liquidate a portfolio once a given level of losses is reached. This practice is called benchmarking, and it uses for portfolios both of equities and derivatives (see Pedersen [11], Basak et al. [2] and Demirer and Lien [3] for more standard stock portfolios, see also Lakshman [9] for other methods and their relative cost). Leoni [10] points out that benchmarking actually achieves the reverse effect than expected; that is, benchmarking actually aggravates the downside of derivative portfolios. There is thus a need to isolate factors, such as appropriate classes of assets, capable of reducing the downside risk of derivative portfolios without involving drastic managerial intervention such as benchmarking. The current paper establishes, through a Monte-Carlo simulation, how the mean-reversion of the 
underlyings dramatically affects the downside risk of derivative portfolios.

Bakshi et al. [1] argues that models where underlyings exhibit meanreversion and stochastic volatility provide the best dynamic hedges and price estimations, even for out-of-sample predictions. The model of Heston [6] provides such an example, and there are large empirical evidence that this model largely outperforms for those purposes standard models such as the Black-Scholes' framework. We focus on Heston's model to carry out a MonteCarlo estimation of the downside risk of a standard management strategy of a derivative portfolio; the point is to estimate how sensitive the downside risk is to a change in mean-reversion of the underlying.

The Monte-Carlo simulation shows that, for every loss level considered in the experiment, the higher the intensity of mean-reversion of the underlyings, the lower the probability of reaching a loss level. This phenomenon is of large statistical significance for large enough loss levels (15\% and above). The second finding is that, for every loss level, the higher the mean-reversion intensity, the longer the expected time to reach those loss levels. Our Monte-Carlo experiment strongly suggests that selecting underlyings with high meanreversion intensity - a property that can be easily detected in statistical tests, is a natural way to reduce the downside risk of derivative portfolios without involving benchmarking and other costly managerial practices.

The basic intuition for this result is that, when exhibiting strong meanreversion effects, the sample paths of the underlyings tend to be more concentrated in a probabilistic sense to the mean of the stochastic process (see Grimmett and Stirzaker [5] Ch. 13 for more on this issue). When dealing 
with risk-neutral dynamics, the mean of the price dynamics for the underlyings is typically the risk-free rate. Therefore, risk-neutral price trajectories of the underlyings are increasingly unlikely to exhibit large and permanent deviations from this rate, as the intensity of mean-reversion increases. Since for most derivatives the extreme payoffs, either positive or negative, are obtained when the underlyings' returns are far off the risk-free return, the reduction in downside risk obtains naturally for most derivatives. The same intuition also shows that, by reducing the downside risk this way, the likelihood of obtaining large positive payoffs is also reduced.

The paper is organized as follows. In Section 2 we describe the experiment; in Section 3 we give the empirical results about the probability of reaching a given loss level as a function of the mean-reversion intensity; in Section 4 we give the empirical results about the expected time of reaching a given loss level as a function of the mean-reversion intensity; Section 4 contains some concluding remarks.

\section{The experiment}

In this section, we describe the model, assumptions and trading strategies that we use to carry out our Monte-Carlo simulation. We first describe four classes of basic options, and the way to form our portfolio with those options. We will then describe our assumptions about the law of motion of the underlyings, which are those described in Heston [6]. We choose this law of motion because it has performed well empirically when pricing derivatives 
as pointed out in Bakshi et al. [1]; it has actually outperformed most of the standard models such as the Geometric Brownian Motions studied in Leoni [10].

\subsection{The options}

The portfolio formation, and classes of options are exactly the same as in Leoni [10], we repeat them for sake of completeness. Even if the experiment is the same up to the choice of the law of motion for the underlyings, the objectives and findings are unrelated to those in the previous reference. We consider 400 different options, which are partitioned into four classes of 100 options each. Every option has a maturity of $T=3$ months, starting with the same common date.

- Class 1. 100 cash-or-nothing options with strike price $K=49$ and end-payment $Q=10$, each of then written on a different underlying. The payoff at time $T$ of the cash-or-nothing option is $Q$ if $S_{T}>K$ and 0 otherwise, where $S_{T}$ is the price of the underlying in 3 months.

- Class 2. 100 lookback options, each of them written on a different underlying. The payoff of a lookback option is $S_{T}-\min (S)$, where $\min (S)$ is the minimal price of the underlying between 0 and $T$.

- Class 3. 100 Asian options, each of them written on a different under-

lying. The payoff of one Asian option is $\max \left\{0, S_{T}-\bar{S}\right\}$, where $\bar{S}$ is the mean of underlying price between 0 and $T$. 
- Class 4. 100 European calls with strike price $K=49$, each of them written on a different underlying. The payoff at the end of the 3 months is $\max \left\{0, S_{T}-K\right\}$.

\subsection{Portfolio formation}

We now describe how our portfolio is formed. The initial wealth of $w_{0}=$ $1,000,000$ is equally allocated among the four classes of options. In every class of options, the wealth allocated to this class is equally distributed across all of those options. That is, if $w_{j}$ is the wealth allocated to Class $j$, then for every option in this class we purchase at current market price, given in Table 2.3 described later so as to match risk-neutral valuation of those assets, a number of contracts whose total value amounts to $w_{j} / 100$ monetary units (we implicitly assume that the options are infinitely indivisible to simplify the analysis, and without any significant loss of generality).

Once the first time horizon (3 months) is reached and the payoffs of all of the options are realized, the proceeds are reinvested in a similar portfolio in the same manner as above. We call a quarter any of such times where options expire and proceeds are reinvested. We consider at most 24 of those quarters, since the results that we obtain in our simulations are all within this horizon.

The fact that options are kept until expiration (or 3 months) in our scenario, instead of being sold before is not restrictive. Indeed, since the current reselling price of the option reflects any loss-gain incurred during the exer- 
cise, the reinvestment of the realized gain-loss into similar assets would not affect the portfolio value since the underlyings follow a Lévy process.

\subsection{Price evolution of underlyings}

In this section, we describe the underlying assets on which the options are written. Before describing the laws of motion of the underlyings, we define $\kappa \in\{e, a, l, c\}$ to be an index denoting the class of options the underlying is assigned to as described earlier, and $j=1, \ldots, 100$ to uniquely describe the option within the class of options $\kappa$. The simulation involves a set of 400 different underlyings, exhibiting 0-pairwise correlation with any other underlyings, whose price processes in a risk-neutral world are described by the following stochastic differential equations

$$
\begin{array}{r}
d S_{t}=I *(r-\delta) S_{t} d t+\sqrt{\nu_{t}} S_{t} d W_{t}^{1}, \\
d \nu_{t}=\left(\alpha-\beta \nu_{t}\right) d t+\sqrt{\nu_{t}} \sigma_{\nu} d W_{t}^{2}
\end{array}
$$

where $S_{t}$ is the price of the underlying at time $t, \nu_{t}>0$ is the instantaneous variance of the underlying assumed to be stochastic, $W_{t}^{i}(i=1,2)$ are independent Brownian motions with law $N(0, t)$ for every time $t$, and $\delta, \beta$, $\sigma_{\nu}$ are positive parameters to be determined later. The variable $I$ captures the intensity of reversion of the variable $S_{t}$ to its mean value $r$ (the risk-free interest rate), in the sense that the higher $I$ the stronger the reversion effect. The variable $I$ will be called the intensity of mean-reversion throughout. Our analysis comes down to observing how an increase in $I$ affects the downside risk of the portfolio formation described earlier. Every underlying 
is assumed to be statistically independent of any other, and thus a more accurate description of the law of motion of those prices should have specified the class of the underlying and its identification within this class; this abuse of notation is meant to simplify the exposition.

This model is taken from Heston [6]. It allows for the volatility of the underlying asset to be randomly determined, and assumes that it follows a Ornstein-Uhlenbeck process (Eq. 2). This model also has the critically important empirical property that stochastic volatility and returns are correlated. The Heston model is much more accurate in describing observed option prices than other standard models such as the Black-Scholes model (see Bakshi [1]); this fact alone justifies our focus on this type of dynamics. Standard empirical findings suggest that the value $\sigma_{\nu}=0.189, \alpha=0.094$, $\beta=12.861$, and $\delta=0.01$ provide the best fit. We will also assume that $r=.05, \nu_{0}=.45$ and the initial stock price is $S_{0}=50$.

We need a discretized version of the continuous-time process described in Eq. (1) and (2) to carry out our numerical simulations. We use the common approximation $W_{t+\Delta t}-W_{t} \approx \epsilon \sqrt{\Delta t}$ for every small enough time variation $\Delta t$, where $\epsilon$ is a random variable with law $N(0,1)$ generating the jumps (see Karatzas and Shreve [8] for a justification). Using the independence of time increments in Brownian motions, both for the prices $S_{t}$ and the instantaneous volatility, the law of motion above can be effectively approximated by 


$$
\begin{array}{r}
S_{t+1}=S_{t}+I *(r-\delta) S_{t} \Delta t+S_{t} \epsilon_{1} \sqrt{\nu_{t} \Delta t} \\
d \nu_{t+1}=\nu_{t}+\left(\alpha-\beta \nu_{t}\right) \Delta t+\epsilon_{2} \sigma_{\nu} \sqrt{\nu_{t} \Delta t}
\end{array}
$$

for every sequence of times $0<t_{0}<\ldots<t_{n}$. We will consider our standard time horizon of $T=3$ months, and we will assume that there are 15 jumps of equal length within those 3 months for every underlying. In order to efficiently simulate the dynamical system described in Eq. (3) and (4), we first need to simulate the stochastic variance in Eq. (4), and then to use the calculated sequence of volatilities into Eq. (3). The underlyings thus differ by the nature of the realized jumps $\epsilon_{i}(i=1,2)$, and those jumps are independent.

Given the previous assumptions, it is now possible to calculate the riskneutral prices of the options described above by using numerical methods. We use a standard Monte-Carlo simulation to calculate the risk-neutral prices of the derivatives, and this simulation is independent of the simulation for the evolution of the portfolio (see Glasserman [4] Chapters 4-5 or Hull [7] Chapter 22 for an introduction to the methods used here). The results are given in Table 1.

\section{Statistical results}

We now give the results of our Monte-Carlo simulations. In a first step, we establish the likelihood of reaching a pre-determined loss level at least once 
Table 1: Monte-Carlo estimations of the risk-neutral prices of the options, as a function of the mean-reversion intensity. Codes are written in $\mathrm{R}$ (see $\mathrm{R}$ project [12]). Figures between brackets are the variances of the estimators. Estimators are calculated with $\mathrm{N}=100,000$ simulations.

\begin{tabular}{||l|cccc||}
\hline Intensity & cash-or-nothing & lookback call & Asian call & European call \\
\hline \hline$I=1$ & 5.236236 & 6.004019 & 3.426517 & 4.463828 \\
& $(0.049288)$ & $(0.059827)$ & $(0.048395)$ & $(0.065198)$ \\
\hline$I=5$ & 5.0668 & 7.471425 & 4.076399 & 5.787401 \\
\hline$I=10$ & $(0.01560)$ & $(0.066783)$ & $(0.052746)$ & $(0.074305)$ \\
& 6.061753 & 9.516904 & 4.953797 & 7.684566 \\
& $(0.048083)$ & $(0.074063)$ & $(0.057426)$ & $(0.08416)$ \\
\hline
\end{tabular}

before the end of the 24 quarters as a function of the intensity of meanreversion. This event would correspond to the activation of a stop-loss strategy, thus a portfolio liquidation as described in Leoni [10], had this strategy been implemented. In a second step, we determine the expected quarter where the previous losses are recorded, again as a function of the intensity of mean-reversion. This exercise allows us to see how critically sensitive the downside risk of derivative portfolios is to an increase in the intensity of mean-reversion. 


\subsection{Failure rate and mean-reversion intensity}

We now turn to describing how the intensity of mean-reversion affects the likelihood of reaching a pre-determined loss level before the end of our horizon of 24 quarters. We define the failure rate of a given simulation to be the number of scenarii where a pre-determined loss level has been reached at least once, divided by the total number of scenarii. The investment scenario has been simulated $N=2500$ times, and Monte-Carlo estimators of activation times are reported for three levels of mean-reversion intensity.

Table 2 below gives the results for three levels of mean-reversion intensity, ( $I=1,5$ and 10$)$, and for various levels of pre-determined losses. The point is to cross-compare the failure rates associated with a given intensity level.

The main result to notice is that, for every loss level, the higher the intensity the lower the failure rate. It turns out that the difference is statistically significant and large for high loss levels (15\% losses and above), although it appears as minor for lower loss levels. For instance, the difference is not statistically significant at $5 \%$ loss level for the intensity levels $I=1$ and $I=5$, but the reduction is statistically significant and important for $I=10$. In contrast, the failure rate is roughly halved at $30 \%$ loss level for the intensity levels $I=5$ and $I=10$, unambiguously showing the major improvement in downside risk reduction when doubling the intensity. It is also surprising to notice that the reduction in downside risk is sensible when switching from $I=1$ to $I=5$, at least for large enough loss levels, but the improvements are largely felt at every loss level only when switching to the highest intensity 
Table 2: Failure rates as a function of the loss level, for various levels of intensity. Figures between brackets are the variances of the estimators. Estimators are calculated with $\mathrm{N}=2500$ simulations.

\begin{tabular}{||l|cccccc||}
\hline \multirow{2}{*}{ Intensity } & \multicolumn{7}{|c||}{ Loss level } \\
& .05 & .1 & .15 & .2 & .25 & .3 \\
\hline \hline$I=1$ & 66.7 & 54.8 & 49.8 & 33.7 & 25.1 & 19 \\
& $(0.942)$ & $(0.994)$ & $(0.992)$ & $(0.944)$ & $(0.942)$ & $(0.784)$ \\
\hline$I=5$ & 66.64 & 53.56 & 40.44 & 28.88 & 22.28 & 14.44 \\
& $(0.95)$ & $(0.96)$ & $(0.95)$ & $(0.832)$ & $(0.703)$ & $(0.703)$ \\
\hline$I=10$ & 59.12 & 41.2 & 29.2 & 19.44 & 13.64 & 7.64 \\
& $(0.95)$ & $(0.94)$ & $(0.908)$ & $(0.79)$ & $(0.78)$ & $(0.53)$ \\
\hline
\end{tabular}

level $I=10$. The numbers are presented in Fig. 1 to have for more intuition.

\subsection{First quarter of failure and intensity}

We now determine the expected first quarter where a given loss level is recorded, for the same mean-reversion intensities as before. This event would correspond to the expected date where a stop-loss strategy would be activated. We consider three intensity levels, $(I=1,5$ and 10), and predetermined loss levels ranging from $5 \%$ to $30 \%$. The first expected quarter where a loss level is reached, together with the variance of those estimators, are given in Table 3 . 
Table 3: Expected first quarter when a given loss level is reached, for various levels of intensity. Figures between brackets are the variances of the estimators. Estimators are calculated with $\mathrm{N}=2500$ simulations.

\begin{tabular}{||l|cccccc||}
\hline \multirow{1}{*}{ Intensity } & \multicolumn{7}{|c||}{ Loss level } \\
& .05 & .1 & .15 & .2 & .25 & .3 \\
\hline \hline$I=1$ & 11.0044 & 14.2504 & 16.8924 & 18.9656 & 20.7284 & 21.7828 \\
& $(0.2)$ & $(0.195)$ & $(0.179)$ & $(0.157)$ & $(0.128)$ & $(0.104)$ \\
\hline$I=5$ & 11.2228 & 14.7172 & 17.4812 & 19.9288 & 21.192 & 22.4344 \\
& $(0.199)$ & $(0.192)$ & $(0.174)$ & $(0.143)$ & $(0.118)$ & $(0.087)$ \\
\hline$I=10$ & 12.4828 & 16.886 & 19.3708 & 21.3964 & 22.3676 & 23.2264 \\
& $(0.205)$ & $(0.185)$ & $(0.156)$ & $(0.117)$ & $(0.092)$ & $(0.061)$ \\
\hline
\end{tabular}

The main result is that the expected quarter of the first hit at a given loss level is an increasing function of the intensity level, for every loss level. That is; the higher the intensity level the longer the expected time needed to reach this loss level. It is important to notice that, for low loss levels inferior to $10 \%$, the time difference is barely statistically significant between $I=1$ and $I=5$. This time difference becomes significant, for every loss level, only when considering $I=10$. When considering losses greater than $15 \%$, the improvement in postponing the first expected time when those losses occur becomes clear when increasing the intensity level. The numbers are presented in Fig. 2 to have more intuition. 


\section{Conclusion}

We have simulated a standard management strategy involving the use of derivatives. The objective of the simulation is to estimate the downside of this management strategy, and to isolate factors capable of reducing this downside risk. The simulations are based on the assumption that the underlyings exhibit mean-reversion, a feature that receive large empirical support.

The main finding is that the higher the intensity of mean-reversion, the lower the probability of reaching a pre-determined loss level. This phenomenon is observed for every loss level that we consider, although it appears of large statistical significance for large loss levels (15\% and above). The sec-

ond finding is that the higher the mean-reversion intensity, the longer the expected time to reach those loss levels. The simulations suggest that selecting underlyings with high mean-reversion effect is a natural way to reduce the downside risk of derivative portfolios.

\section{References}

[1] Bakshi, G., Cao, C. and Z. Chen. (1997) "Empirical Performance of Alternative Option Pricing Models, Journal of Finance, 52, 2003- 2049.

[2] Basak, S., Shapiro, A. and L. Tepla (2006) "Risk management with benchmarking." Management Science 52, 542-557.

[3] Demirer, R. and D. Lienb (2003) "Downside risk for short and long hedgers." International Review of Economics \& Finance 12, 25-44. 
[4] Glasserman, P. (2004) Monte-Carlo Methods in Financial Engineering. New-York: Springer Science.

[5] Grimmett, G. and D. Stirzaker (2006) Probability and Random Processes. Oxford: Oxford University Press.

[6] Heston, S. (1993) "A Closed-Form Solution for Options with Stochastic Volatility, with Applications to Bonds and Currency Options," Review of Financial Studies 6, 327-343.

[7] Hull, J. (2006) Options, Futures and Other Derivatives. (6th ed.) Upper Saddle River: Prentice Hall.

[8] Karatzas, I. and S. Shreve (2001) Brownian Motion and Stochastic Calculus. New-York: Springer Science.

[9] Lakshman, A. (2008) "An option pricing approach to the estimation of downside risk: A European cross-country study." Journal of Derivatives 8 Hedge Funds 14, 31-41.

[10] Leoni, P. (2008) "Monte-Carlo estimations of the downside risk of derivative portfolios," IEEE Proceedings of the 4th Conference on Wireless Communications, Networking and Mobile Computing (2008), 1-5 (DOI: 10.1109/WiCom.2008.2273).

[11] Pedersen, C. (2001) "Derivatives and downside risk." Derivatives Use, Trades and Regulations 7, 251-268. 
[12] R Project. (2006) Statistical software. [Online]. Available: http://www.r-project.org

[13] Shen, S. and A. Wangb (2001) "On stop-loss strategies for stock investments." Applied Mathematics and Computation 119, 317-337. 


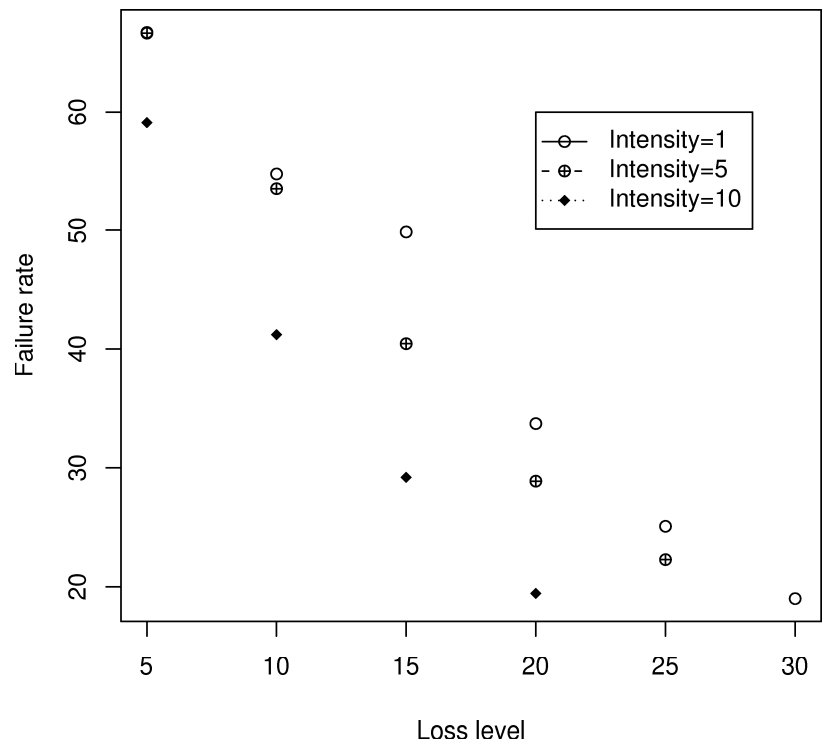

Figure 1: Failure rates as a function of the mean-reversion intensity, for various levels of loss. 


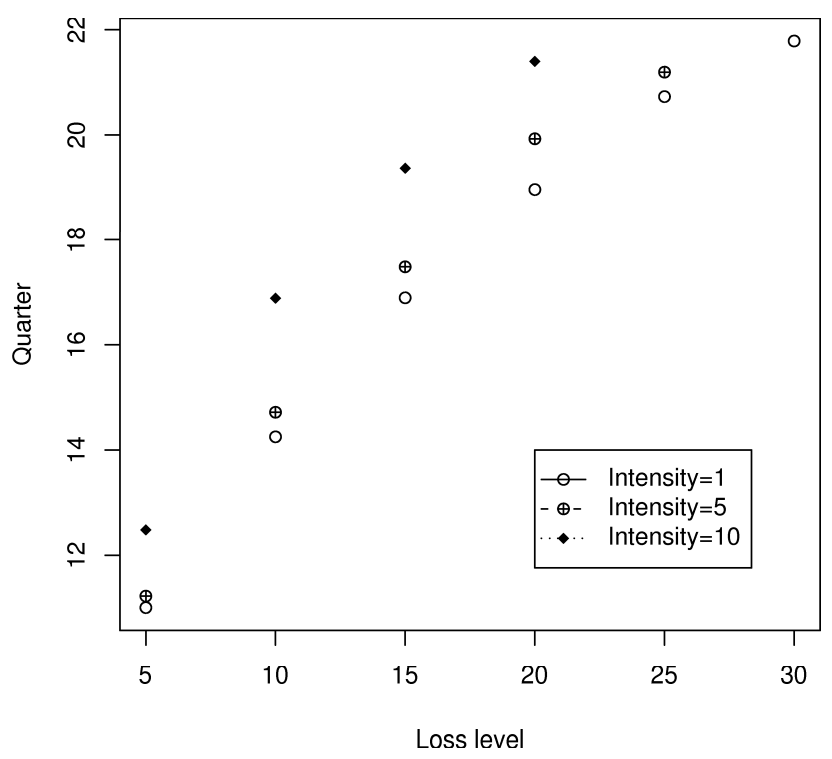

Figure 2: Expected first quarter when a given loss level is reached, for various levels of intensity. 